\title{
Implante de Múltiplos Stents Farmacológicos para o Tratamento da Doença Multiarterial em Paciente Diabética
}

\author{
Galo Maldonado', Arturo Ricardo Quizhpe', José de Ribamar Costa Jr1, Marinella Centemero', \\ Alexandre Abizaid ${ }^{1}$, Fausto Feres ${ }^{1}$, Ricardo Costa ${ }^{1}$, Rodolfo Staico ${ }^{1}$, Luiz Alberto Mattos ${ }^{1}$, Áurea Chaves ${ }^{1}$, \\ Julio Paiva', Paulo Márcio Nunes' ${ }^{1}$ Amanda MR Sousa', J. Eduardo Moraes Rego Sousa ${ }^{1}$
}

\begin{abstract}
RESUMO
A incidência de diabetes mellitus tem aumentado continuamente nos últimos anos. Os pacientes acometidos por esta enfermidade crônica têm maior risco de desenvolver doença aterosclerótica coronária. Infelizmente, devido ao freqüente envolvimento difuso de múltiplos vasos, a intervenção percutânea, quer seja com cateter-balão ou com stent não farmacológico, tem sido associada a pior prognóstico quando comparada à cirurgia de revascularização miocárdica neste grupo de pacientes. Recentemente, a introdução dos stents farmacológicos renovou o entusiasmo para o tratamento percutâneo de pacientes com lesões mais complexas. Neste artigo, reportamos um caso de uma paciente de 63 anos, portadora de diabetes em uso de insulina, tratada percutaneamente com nove stents farmacológicos (Taxus ${ }^{\mathrm{TM}}$, Boston Scientific).
\end{abstract}

DESCRITORES: Coronariopatia. Diabetes mellitus. Contenedores.

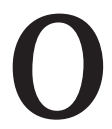
diabetes mellitus (DM) é uma doença crônica, cuja incidência vem aumentando notavelmente no mundo inteiro, constituindo na atualidade um importante problema de saúde pública.

Os pacientes diabéticos são mais propensos ao desenvolvimento de doença coronária, sendo esta a principal causa de internação hospitalar nesta população $(75 \%)$. As complicações cardiovasculares são responsáveis por $80 \%$ dos óbitos entre estes pacientes ${ }^{1}$.

A revascularização miocárdica, cirúrgica ou percutânea, representa um desafio maior neste subgrupo de doentes, uma vez que os portadores de DM são fre-

\footnotetext{
1 Instituto Dante Pazzanese de Cardiologia, São Paulo, SP, Brasil. Correspondência: Galo Maldonado. Serviço de Cardiologia InvasivaInstituto Dante Pazzanese de Cardiologia. Av. Dante Pazzanese, 500 - Ibirapuera - São Paulo - SP - Caixa Postal 215

E-mail: galomaldonado@uol.com.br

Recebido em: 12/1/2007 • Aceito em: 4/5/2007
}

\section{SUMMARY}

Use of Multiple Drug-Eluting Stents for the Treatment of Multivessel Disease in a Diabetic Patient

The incidence of diabetes mellitus has continuously risen over recent years. Patients with this chronic condition have a higher risk of developing coronary artery disease. Unfortunately, due to the frequent diffuse involvement of multiple vessels, the long term outcomes after percutaneous intervention, whether by balloon catheter or bare-metal stent, has always been poor and frequently inferior to cardiac surgery for this subset of patients. Recently, the introduction of drug-eluting stents (DES) has brought new hope to treat patients with more complex diseases percutaneously. In this paper we report a successful percutaneous approach of a 63-year-old insulin dependent diabetic patient treated with nine DES (Taxus ${ }^{\mathrm{TM}}$, Boston Scientific).

DESCRIPTORS: Coronary disease. Diabetes mellitus. Stents.

qüentemente acometidos de lesões difusas em múltiplos vasos, fazendo com que qualquer das duas técnicas atualmente disponíveis tenham resultados, a longo prazo, menos favoráveis comparados aos não-diabéticos.

Até recentemente, quando os catéteres-balão e os stents não farmacológicos constituíam-se nas mais efetivas armas do arsenal terapêutico percutâneo, havia evidências, ainda que não definitivas, de que a cirurgia de revascularização miocárdica era mais efetiva para tratar pacientes diabéticos ${ }^{2}$. Todavia, o uso de terapia adjunta antiplaquetária mais eficaz e a incorporação dos stents farmacológicos no tratamento de lesões mais complexas têm melhorado, significativamente, os resultados da intervenção coronária percutânea (ICP), em diabéticos. A seguir, apresentamos o caso de uma paciente diabética submetida a ICP com implante de múltiplos stents farmacológicos e incluída no estudo "FREEDOM" (Future Revascularization Evaluation in Patients with Diabetes Mellitus: Optimal Management of Multivessel Disease). 


\section{RELATO DO CASO}

\section{Histórico Clínico}

Paciente do sexo feminino, de 63 anos, diabética em uso de insulina há dois anos, ex-tabagista, hipertensa, dislipêmica e com história de angina estável classe funcional I (classificação da Canadian Cadiovascular Society) há 8 meses, com rápida progressão para classe III nos últimos 2 meses anteriores ao procedimento.

Foi avaliada por cardiologista clínico que solicitou teste ergométrico, positivo para isquemia, devido a infradesnível do segmento ST de 1,5 $\mathrm{mm}$ aos 3 minutos de exercício, associado a dor precordial típica. Frente a esses resultados, optou-se por estratificação invasiva por meio de cateterismo diagnóstico.

\section{Cinecoronariografia}

O exame evidenciou doença multiarterial, estando a artéria coronária direita (CD) ocluída em seu 1/3 médio, com opacificação de seu leito distal por circulação intracoronária grau II. A artéria descendente anterior esquerda (DA) apresentava lesões múltiplas, difusas e graves em todos os seus segmentos, e a artéria circunflexa (CX) exibia duas lesões importantes no seu segundo ramo marginal, no 1/3 médio e distal (Figuras 1 a 3). A função ventricular esquerda estava preservada.

\section{Procedimento}

Logo após a discussão do caso em reunião clínicocirúrgica, e da consideração com a paciente sobre as opções de tratamento, optou-se por incluí-la no protocolo multicêntrico "FREEDOM". Este caso foi randomizado para ICP com a utilização de stents com liberação de paclitaxel (Taxus $^{\text {TM }}$ Liberté Stent, Boston Scientific Corp.). Antes de iniciar o procedimento, foi administrado Tirofiban (Agrastat ${ }^{\circledR}$, Merck Sharp \& Dohme) intravenoso, de acordo com orientação protocolar. Para a intervenção da CD, foi utilizado um cateter-guia Amplatz de direita e um fio-guia PT2 ${ }^{\mathrm{TM}}$ (Boston Scientific Corp.), o qual ultrapassou a lesão com ajuda de um balão Maverick ${ }^{\circledR}$ 2,0x9 mm (Boston Scientific Corp.). A lesão foi pré-dilatada em vários segmentos e, a seguir, implantaram-se três stents Taxus ${ }^{\mathrm{TM}}(2,5 \times 24$ mm, 2,5x20 mm e 2,75x $32 \mathrm{~mm}$ ), todos insuflados com alta pressão e com "overlapping", recobrindo a CD em praticamente toda sua extensão ("full metal jacket").

A artéria DA foi abordada com uso de um cateterguia Judkins de esquerda e o mesmo fio-guia acima mencionado. Realizou-se pré-dilatação com o mesmo cateter-balão previamente utilizado para o mesmo procedimento na CD. A seguir, quatro stents Taxus ${ }^{\mathrm{TM}}$ foram implantados $(2,25 \times 12 \mathrm{~mm}, 2,25 \times 12 \mathrm{~mm}, 2,5 \times 12 \mathrm{~mm}$ e 2,75x24 mm), sem zonas de "overlapping", uma vez que a distância entre as lesões era superior a $5 \mathrm{~mm}$. Após o implante do sétimo stent, foi tratado o segundo ramo marginal da artéria CX com a utilização dos mesmos cateter e fio-guia previamente descritos para o tratamento da artéria DA. Realizou-se pré-dilatação da lesão mais distal e, a seguir, implantaram-se mais dois stents Taxus ${ }^{\mathrm{TM}}(2,25 \times 12 \mathrm{~mm}$ e $2,75 \times 16 \mathrm{~mm})$, sem "overlapping".

O procedimento transcorreu sem intercorrências, com duração de duas horas e utilização de $350 \mathrm{ml}$ de contraste de baixa osmolaridade (Hexabrix, Guerbet). O resultado final é mostrado nas Figuras 1 a 3 .

\section{Seguimento clínico}

A paciente evoluiu sem complicações após a intervenção, recebendo alta hospitalar $48 \mathrm{~h}$ após o procedimento, em boas condições clínicas, sem alteração da função renal e dos marcadores cardíacos (CPK, CKMB e troponina I). Foi prescrita dupla terapia antiplaquetária (clopidogrel e AAS) por 12 meses, além de sinvastatina, atenolol, captopril e insulina subcutânea. No retorno, 30 dias após a ICP, a paciente mantevese assintomática e em ótimas condições clínicas. Na atualidade (três meses de evolução), a paciente não refere sintomatologia compatível com recorrência de seus sintomas prévios.

\section{DISCUSSÃO}

O caso acima descrito, ainda que se constitua em uma exceção do ponto de vista terapêutico, representa a manifestação clínica e angiográfica mais freqüente da doença aterosclerótica entre os portadores de DM, com acometimento múltiplo e difuso da circulação coronária.

A associação de diabetes e doença difusa, vasos de fino calibre, acometimento multiarterial e leito coronário distal ruim, contribui para elevar a dificuldade técnica da ICP, e, sobretudo, para obtenção de resultados clínicos desfavoráveis no seguimento tardio após as terapias de revascularização, com maiores taxas de recorrência da doença nos sítios previamente tratados, quando comparados aos pacientes não diabéticos.

Estima-se que, na atualidade, $20 \%$ a $30 \%$ de todos os procedimentos de revascularização sejam realizados em diabéticos ${ }^{3}$. Porém, ainda não há consenso sobre a melhor estratégia para abordar estes pacientes.

No começo da década de oitenta, foram realizados vários estudos randomizados comparando as duas estratégias de revascularização então disponíveis: a cirurgia de revascularização miocárdica (RM) versus angioplastia com cateter-balão (ATC). Dentre eles, destacou-se o estudo BARI, que demonstrou que, ao fim de cinco anos de evolução, o uso de ATC em pacientes diabéticos multiarteriais tinha piores resultados clínicos, com maiores taxas de eventos maiores, quando comparado ao tratamento cirúrgico ${ }^{2}$. Especula-se que a alta taxa de patência do enxerto de artéria mamária esquerda para a artéria DA conferiu uma proteção para a parede anterior do ventrículo esquerdo, fato não observado entre os pacientes submetidos à ICP com balão. 
Maldonado G, et al. Implante de Múltiplos Stents Farmacológicos para o Tratamento da Doença Multiarterial em Paciente Diabética. Rev Bras Cardiol Invas 2007; 15(3): 302-306.

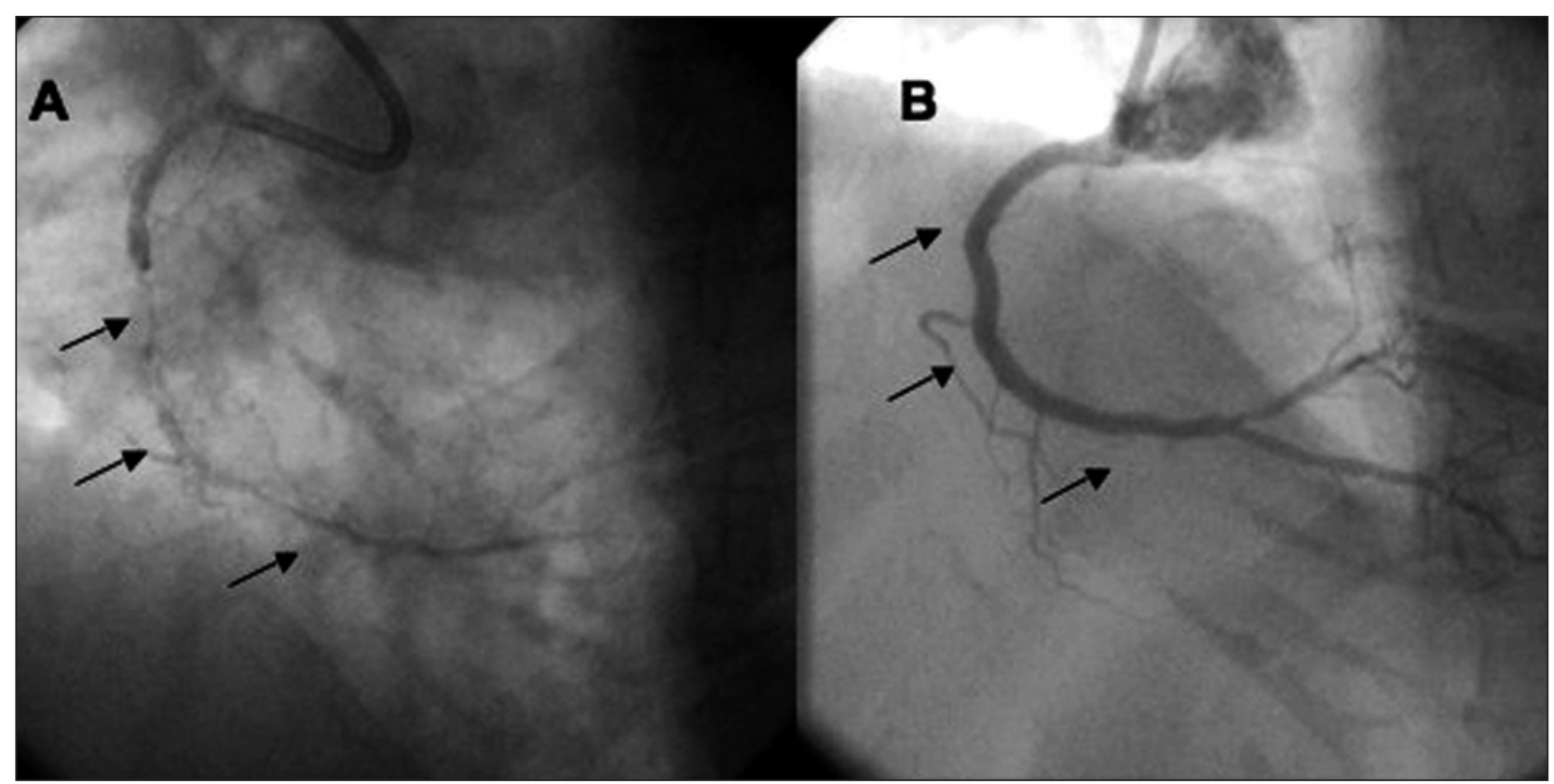

Figura 1 - Artéria coronária direita pré e pós-angioplastia. No painel A, as setas apontam para o acometimento aterosclerótico difuso do 1/3 médio e distal da CD. No painel B, visualizamos o resultado final após implante de três stents Taxus ${ }^{T M} L_{\text {Liberté. }}$

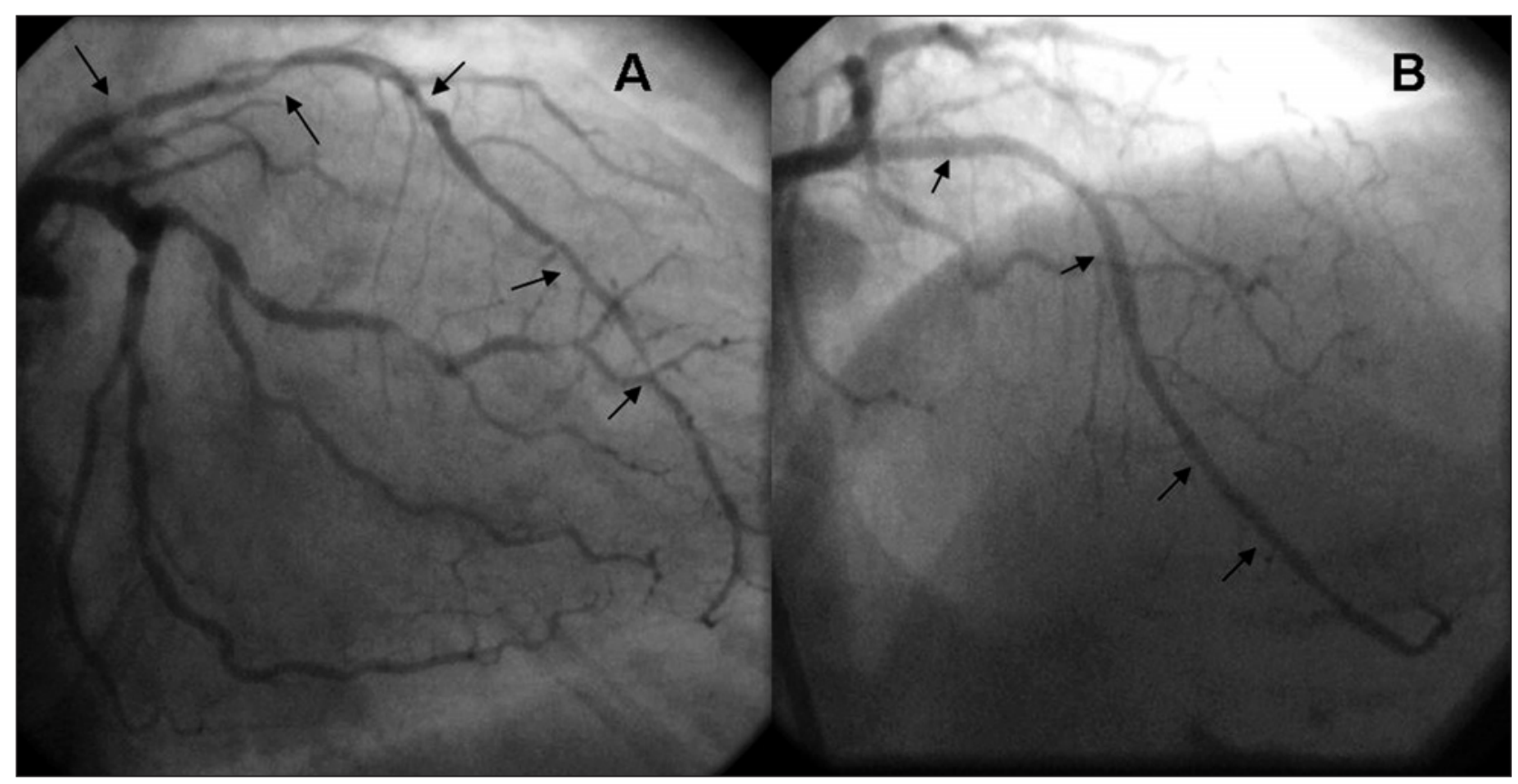

Figura 2 - Artéria descendente anterior esquerda pré e pós-angioplastia. No painel A, observamos presença de múltiplas lesões obstrutivas acometendo $1 / 3$ proximal, médio e distal da artéria DA. O painel B apresenta o resultado final após implante de quatro stents Taxus ${ }^{\text {TM }}$ Liberté (setas), sem "overlapping".

Em geral, as duas estratégias de revascularização não diferem quanto à mortalidade ou ao infarto agudo do miocárdio. Porém, quando analisamos a necessidade de novo procedimento de revascularização, a cirurgia tem nítida vantagem sobre a ICP com cateter-balão e mesmo com stents não-farmacológicos ${ }^{4-7}$.
Entre os pacientes com DM, a situação é diferente do contexto geral, tendo a cirurgia de RM não só diminuído a necessidade de novos procedimentos de revascularização, mas também reduzido a mortalidade no seguimento clínico tardio, quando comparada à ICP com balão e stents não-farmacológicos ${ }^{2,4}$. 


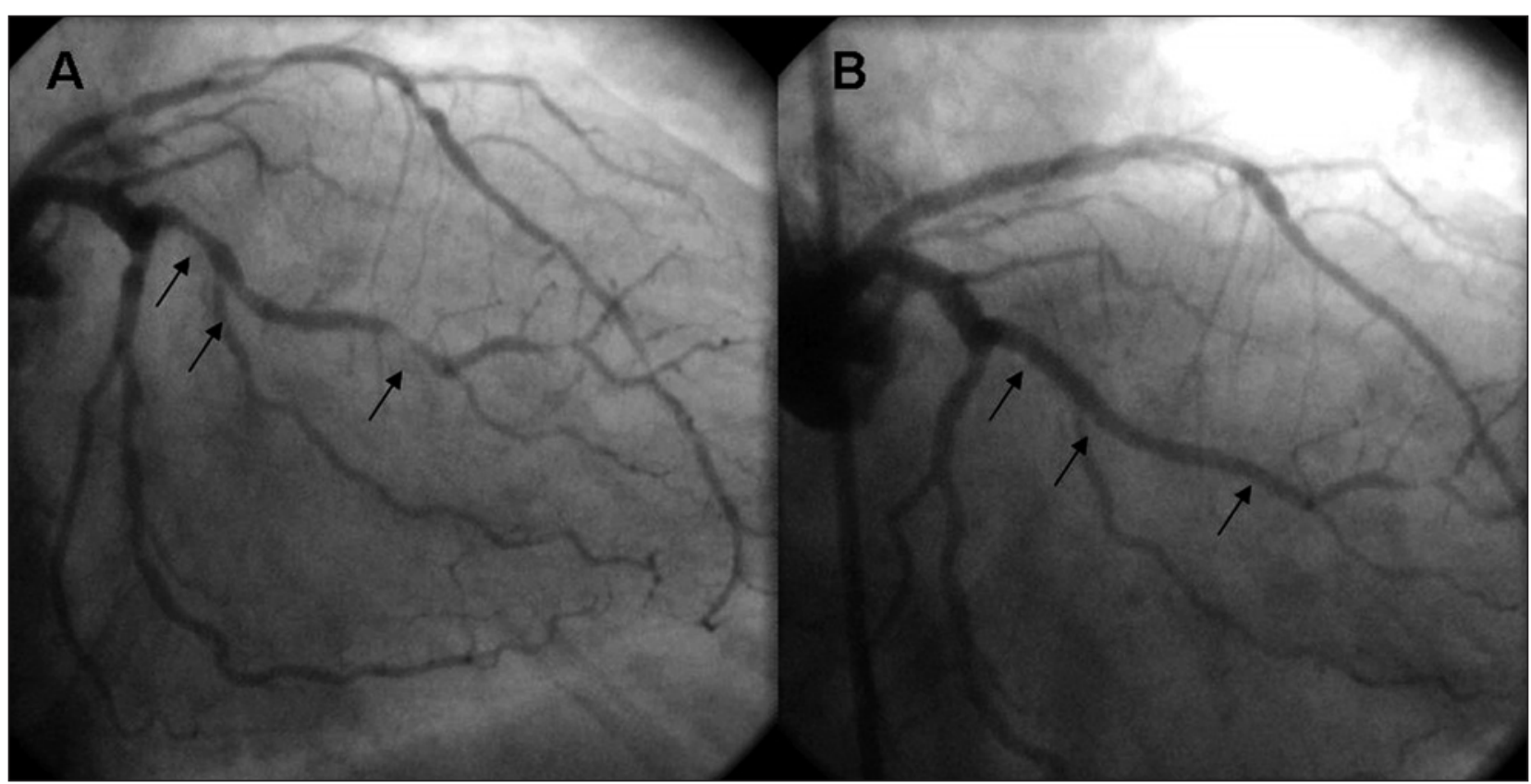

Figura 3 - Ramo marginal da artéria circunflexa pré e pós-angioplastia. Observa-se acometimento do 1/3 proximal e médio do grande ramo marginal da artéria CX (setas, painel A). No painel B, observamos o excelente resultado final da intervenção após implante de dois stent Taxus ${ }^{\mathrm{TM}}$ Liberté.

Uma recente meta-análise de quatro estudos randomizados com uso de stents não-farmacológicos versus cirurgia de RM demonstrou uma diferença, a favor do grupo cirúrgico, de $11 \%$ na incidência de eventos combinados. Esta superioridade da cirurgia deveu-se, sobretudo, à necessidade de novo procedimento de revascularização no grupo tratado com $\mathrm{ICP}^{8}$.

$\mathrm{Na}$ era dos stents farmacológicos, o tratamento dos pacientes diabéticos e multiarteriais tem merecido especial atenção. Neste contexto, o registro RESEARCH incluiu 338 pacientes com doença coronária multiarterial tratados com stents com liberação de sirolimus ${ }^{9}$, dos quais 94\% tiveram uma sobrevivência livre de eventos ao fim de um ano de evolução clínica. Mais recentemente, Serruys ${ }^{10}$ apresentou os resultados do estudo ARTS II, que comparou pacientes tratados com stents com liberação de sirolimus a um grupo controle histórico constituído pelos pacientes randomizados para cirurgia e stent não farmacológico do estudo ARTS I. Demonstrou-se, pela primeira vez, que a sobrevivência livre de eventos no grupo sirolimus foi superior a dos indivíduos tratados com stents não farmacológicos e com cirurgia de $\mathrm{RM}^{10}$. No estudo TAXUS $\mathrm{V}^{11}$, foi testado o stent liberador de paclitaxel em lesões de alta complexidade (vasos finos e lesões longas), com uma alta porcentagem de pacientes diabéticos (30\%). Os resultados mostraram que a necessidade de revascularização da lesão-alvo foi de $8 \%$.

$\mathrm{Na}$ atualidade, encontram-se em fase de randomização dois estudos de grande porte - FREEDOM e CARDia - comparando ICP com uso dos stents farma- cológicos à cirurgia de RM em pacientes diabéticos multiarteriais. Além de comparar as duas estratégias de tratamento do ponto de vista de resultados clínicos, estes estudos ajudarão também a definir a questão de custo-efetividade na abordagem destes doentes. Cabe reforçar que o caso aqui apresentado, embora com excelente resultado clínico a curto prazo, do ponto de vista econômico está longe de constituir-se na terapêutica ideal a ser instituída para pacientes multiarteriais, sobretudo em países em desenvolvimento. Por fim, é importante ressaltar que a presença de diabetes mellitus tem sido implicada na ocorrência de trombose de stents farmacológicos ${ }^{12}$ e que ainda não está bem definido o período de tempo que pacientes diabéticos submetidos a implante de múltiplas endopróteses necessitam fazer uso de terapia antiplaquetária combinada (AAS + Tienopiridínico). O paciente do caso aqui descrito fará uso de AAS e Clopidogrel por um período mínimo de 12 meses, conforme protocolo do estudo FREEDOM.

Entretanto, independente dos achados destes estudos, estamos certos que a cardiologia intervencionista continuará procurando novos dispositivos e terapias farmacológicas adjuntas para oferecer aos pacientes diabéticos uma opção de tratamento mais segura e com melhores resultados em longo prazo.

\section{REFERÊNCIAS BIBLIOGRÁFICAS}

1. Kannel WB, McGee DL. Diabetes and cardiovascular disease. The Framingham study. JAMA. 1979;241(19):2035-8.

2. Comparison of coronary bypass surgery with angioplasty in patients with multivessel disease. The Bypass Angioplasty 
Revascularization Investigation (BARI) Investigators. N Engl J Med. 1996;335(4):217-25.

3. Kip KE, Faxon DP, Detre KM, Yeh W, Kelsey SF, Currier JW. Coronary angioplasty in diabetic patients. The National Heart, Lung, and Blood Institute Percutaneous Transluminal Coronary Angioplasty Registry. Circulation. 1996;94(8):1818-25.

4. Abizaid A, Costa MA, Centemero M, Abizaid AS, Legrand VM, Limet RV, et al. Clinical and economic impact of diabetes mellitus on percutaneous and surgical treatment of multivessel coronary disease patients: insights from the Arterial Revascularization Therapy Study (ARTS) trial. Circulation. 2001;104(5):533-8.

5. Rodriguez A, Bernardi V, Navia J, Baldi J, Grinfeld L, Martinez J, et al. Argentine Randomized Study. Coronary Angioplasty with Stenting versus Coronary Bypass Surgery in patients with Multi-Vessel Diasease (ERACI II): 30-day and one-year follow-up results. ERACI II Investigators. J Am Coll Cardiol. 2001;37(1):51-8.

6. SOS Investigators. Coronary artery bypass surgery versus percutaneous coronary intervention with stent implantation in patients with multivessel coronary artery disease (the Stent or Surgery trial): a randomised controlled trial. Lancet. 2002;360(9338):965-70.

7. Hueb W, Soares PR, Gersh BJ, César LA, Luz PL, Puig LB, et al. The Medicine, Angioplasty, or Surgery Study (MASSII): a randomized, controlled clinical trial of three therapeutic strategies for multivessel coronary artery disease: one-year results. J Am Coll Cardiol. 2004;43(10):1743-51.

8. Mercado N. Coronary artery bypass surgery versus stenting for the treatment of multivessel disease: meta-analysis of ARTS-1, SoS, ERACl-2, and MASS-2. Disponível em: www.tctmd.com

9. Lemos PA, Serruys PW, van Domburg RT, Saia F, Arampatzis CA, Hoye A, et al. Unrestricted utilization of sirolimuseluting stents compared with conventional bare stent implantation in the "real world": the Rapamycin-Eluting Stent Evaluated At Rotterdam Cardiology Hospital (RESEARCH) registry. Circulation. 2004;109(2):190-5.

10. Serruys PW. Arterial revascularization therapies study part II of the sirolimus-eluting bx velocity ${ }^{\circledast}$ balloon expandable stent in the treatment of patients with multivessel de novo coronary artery lesions. TCT 2004; USA. Disponível em: www.tctmed.com

11. Stone GW, Ellis SG, Cannon L, Mann JT, Greenberg JD, Spriggs D, et al. Comparison of a polymer-based paclitaxeleluting stent with a bare metal stent in patients with complex coronary artery disease: a randomized controlled trial. JAMA. 2005;294(10):1215-23.

12. lakovou I, Schmidt T, Bonizzoni E, Ge L, Sangiorgi GM, Stankovic G, et al. Incidence, predictors, and outcome of thrombosis after successful implantation of drug-eluting stents. JAMA. 2005;293(17):2126-30. 